This item was submitted to Loughborough's Research Repository by the author.

Items in Figshare are protected by copyright, with all rights reserved, unless otherwise indicated.

\title{
Hydrostatic cyclic expansion extrusion (HCEE) as a novel severe plastic deformation process for producing long nanostructured metals
}

PLEASE CITE THE PUBLISHED VERSION

https://doi.org/10.1016/j.msea.2018.01.116

PUBLISHER

(C) Elsevier

VERSION

AM (Accepted Manuscript)

\section{PUBLISHER STATEMENT}

This work is made available according to the conditions of the Creative Commons Attribution-NonCommercialNoDerivatives 4.0 International (CC BY-NC-ND 4.0) licence. Full details of this licence are available at: https://creativecommons.org/licenses/by-nc-nd/4.0/

\section{LICENCE}

CC BY-NC-ND 4.0

\section{REPOSITORY RECORD}

Samadpour, F., Ghader Faraji, P. Babaie, Rickie Bewsher, and Mahdi Mohammadpour. 2019. "Hydrostatic Cyclic Expansion Extrusion (HCEE) as a Novel Severe Plastic Deformation Process for Producing Long Nanostructured Metals". figshare. https://hdl.handle.net/2134/28370. 


\title{
Hydrostatic cyclic expansion extrusion (HCEE) as a novel severe plastic deformation process for producing long nanostructured metals
}

\author{
F. Samadpour ${ }^{\mathrm{a}}$, G. Faraji ${ }^{* a}$, P. Babaie ${ }^{\mathrm{a}}$, S. R. Bewsher ${ }^{\mathrm{b}}$, M. Mohammadpour ${ }^{\mathrm{b}}$ \\ ${ }^{a}$ School of Mechanical Engineering, College of Engineering, University of Tehran, Tehran, 11155-4563, Iran. \\ ${ }^{b}$ Wolfson School of Mechanical, Electrical \& Manufacturing Engineering, Loughborough University, \\ Loughborough, UK \\ *Corresponding author, email: ghfaraji@ut.ac.ir, Tel/Fax: +982161119966.
}

\begin{abstract}
In this paper, hydrostatic cyclic expansion extrusion (HCEE) is developed as a new severe plastic deformation technique for processing of the relatively longer ultrafine grained samples. Increasing the length of the processed sample, decreasing the processing load astonishingly, and increasing the hydrostatic stresses are the main advantages of HCEE. In This process lubricant surrounded around workplaces plays the main role in reducing the friction load and increase pressure hydrostatic. HCEE process was executed to commercial pure aluminum 1050 at room temperature, and microstructural evolution and the mechanical properties were examined. Microstructure evolution of this process was investigated by transmission electron microscopy (TEM) and back-scattered diffraction (EBSD). TEM and EBSD revealed an ultrafine grained microstructure after the two passes of the HCEE process. The average size of grains and subgrain decreased from $50 \mu \mathrm{m}$ in the annealed sample to $0.76 \mu \mathrm{m}$ after two passes of the process. Mechanical properties such strength and hardness improved because of the large effect strain. Yield and ultimate strength were increased from $40 \mathrm{MPa}$ and $52 \mathrm{MPa}$ to $109 \mathrm{MPa}$ and $115 \mathrm{MPa}$ just after one pass of HCEE process, also Microhardness was increased from $36 \mathrm{HV}$ to $45 \mathrm{HV}$ at first passes.
\end{abstract}

Keywords: Severe plastic deformation, Hydrostatic Cyclic Expansion-Extrusion, Scale-up, electron backscattering diffraction (EBSD), Grain refinement.

\section{Introduction}


In the last two decades, research in the field of severe plastic deformation (SPD) has been developing rapidly. Ultrafine Grained (UFG) and Nano Grained (NG) materials obtained from SPD have shown superior and unique mechanical and physical properties [1, 2]. The most common SPD techniques for processing bulk materials are equal channel angular pressing (ECAP) [3, 4], cyclic extrusion compression (CEC)[5], accumulative roll bonding (ARB) [6], and high-pressure torsion (HPT) [7, 8]. The CEC method was invented in 1986 for unlimited deformation of metals and alloys [9] which was named "reciprocating extrusion" in some publications $[10,11]$. The CEC method was invented to allow arbitrarily large strain deformation of a sample with the preservation of the original sample shape [12-15]. However, due to the complication of the die set up for CEC, a precise back pressure system which acts in synchronization with the primary punch is required. To solve the back pressure problem in CEC, Cyclic Expansion Extrusion (CEE) as a modified counterpart of CEC was introduced by Pardis et al. in 2011 [16]. The force needed to extrude the material is supposed to provide a proper amount of back pressure for the expansion [17, 18]. Needing complicated back pressure system does not exist in the CEE method, and therefore, the CEE technique has good potential for SPD processing. However, in both CEC and CEE processes, by increasing the workpiece length, friction force increases sharply while the deformation part of the total force is constant, so the total force increases dramatically. Thus, the punch deforms, yields or buckles under high forces and the process cannot be completed. This would be the major setback of the method, remaining at a laboratory scale. Until now, the maximum length/diameter ratios of CEE or CEC samples are below 5, making these processes unsuitable for industrial applications [19, 20], moreover, most spd techniques such CEE cause heat to form due to deformation of the plastic, but this method maybe prevent heat and dynamic recovery in the process due to the presence of fluid. In addition, strain rate can more than CEE process because friction force is very little, so times of process is less than other techniq[21, 22]. this method can make longer and refiner grain sample.

The present study aims to introduce an efficient technique based on CEE to make the possibility for processing large samples. This paper proposes a new method entitled Hydrostatic Cyclic Expansion Extrusion (HCEE) for producing ultrafine grained and nanostructured samples in relatively long length.

\section{Principles of HCEE}


A schematic of HCEE process, patented in 2017 by the current authors [23], is shown in Fig. 1. The main difference between HCEE and conventional CEE process is the using of fluid pressure instead of frictional contact. As a result, the friction force is almost removed. As can be seen in Fig. 1a, a hydraulic fluid fills the space between the initial billet and the primary pressure container. The fluid is pressurized and sealed by a movable punch and an especial designed PTFE polymeric seal. The HCEE process consists of four steps, and the sequences are shown in Fig. 1. At first, the initial billet is placed into the die, and then hydraulic fluid fills the space between the billet and primary pressure chamber. A movable punch and polymeric seal are used to increase fluid pressure to extrude the billet. The initial billet is forced to move down by the pressurized fluid to reach the bottom of the punch. The billet will be expanded to fill the die cavity which is a deformation zone. Then, the bottom punch is removed, and the first pass is completed after transferring the billet through the chamber. To perform the second pass, the die rotates $180^{\circ}$, and the process is repeated the same as before. This technique can be done to achieve a required number of passes. The equivalent plastic strain is similar to conventional CEE which can be calculated for each cycle of HCEE from:

$$
\bar{\varepsilon}=4 \ln \frac{D}{d}
$$

Therefore, from Eq. 1 the total accumulated strain per each pass of HCEE considering the die parameters used here could be estimated to be about 1.34.

\section{Experimental and FEM procedures}

A commercially pure Al 1050 (99.5\%) was used in this study. All samples were machined to a diameter of $10 \mathrm{~mm}$ and a length of a $100 \mathrm{~mm}$. They were annealed at the temperature of $350{ }^{\circ} \mathrm{C}$ for 2 hours and cooled in the furnace[24]. The die and its components, as shown in Fig. 1e, were manufactured from hot worked tool steel and hardened to 55 HRC. The seal was made of a polymeric composite containing metal particles with a slightly larger diameter compared to the initial channel with the aim of preventing the fluid from leaking. Geometric die parameters are $\mathrm{D}=14 \mathrm{~mm}, \mathrm{~d}=10 \mathrm{~mm}, d_{0}=12 \mathrm{~mm}, \mathrm{~L}=1 \mathrm{~mm}, \mathrm{r}=3 \mathrm{~mm}$ and $\alpha$ $=60^{\circ}$ as shown in Fig.1a. The process was conducted using a hydraulic press at a ram speed of about $5 \mathrm{~mm} / \mathrm{min}$. Microhardness and tensile tests were conducted to investigate mechanical properties of the HCCE specimen. The tensile tests were carried out at room temperature with an initial strain rate $10^{-3} \mathrm{~s}^{-1}$. 
The Vickers microhardness testing was performed using an indenter load of $100 \mathrm{gr}$ and a loading time of $10 \mathrm{sec}$. The microstructure of annealed samples is evaluated using optical microscopy (OM) after electro polishing. The HCEE processed samples was evaluated using transmission electron microscope (TEM) after preparing a thin lamella samples with the sizes of $5 \times 8 \mu^{2}$ from the mid radius of the cross section of the processed specimen using the in-situ lift-out procedure on a dual beam system (FEI Nova Nanolab 600). Due to the limited number of grains observed in the prepared lamella, characterization of a broader area was desirable in order to obtain more conclusive results. Preparation of larger lamella would face the practical challenges of sample distorsion during the lift-out process. Hence, a Field Emission Gun Scanning Electron Microscope (JEOL 7100F FEGSEM) equipped with an Electron Backscatter Diffraction (EBSD) camera (EDAX TSL) has been utilized under conditions of $20 \mathrm{kV}$ and $\sim 25 \mathrm{nA}$. The final polishing of the sample has been performed using a "VibroMat 2" vibratory polishing machine with no load. The microstructure has been post-processed using Oxford Instruments AZtec software package. The large area EBSD scanning provided concludable results. Finite element simulations were carried out using commercial software Abaqus\Explicit in the form of axisymmetric 2D. In the contact between the die and fluid, simulation is considered frictionless. Where the die chamber and sample have a contact at the deformation zone, the friction coefficient is considered to be 0.08 [25]. 


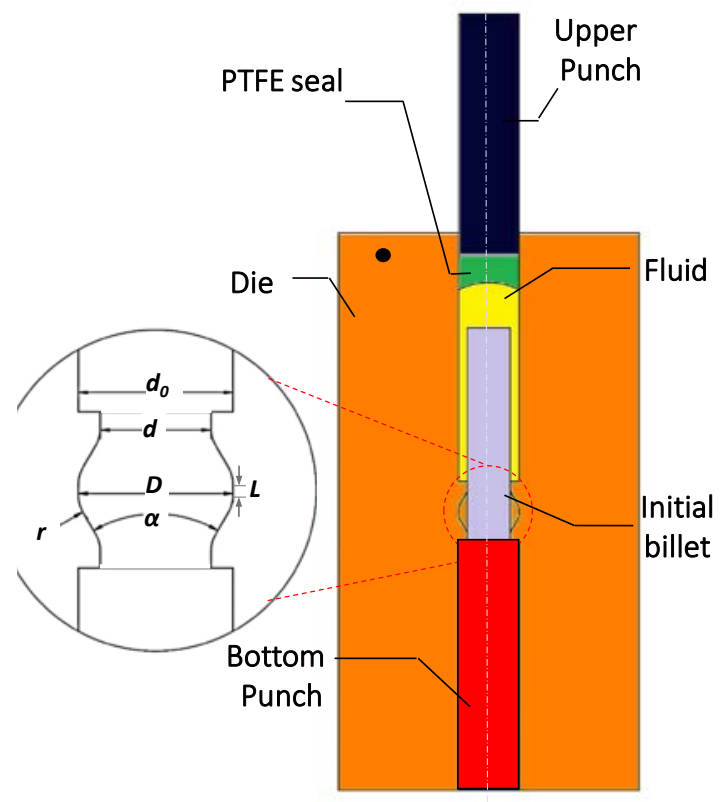

(a)

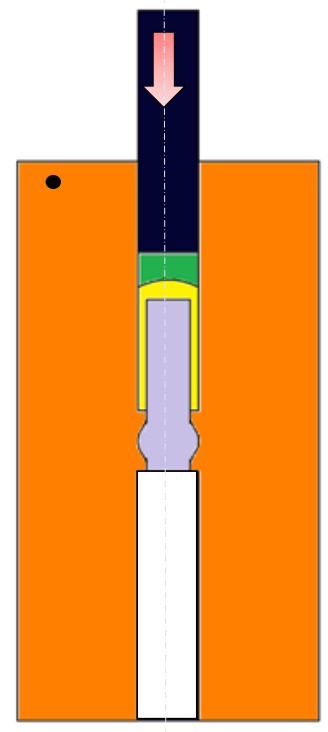

(b)

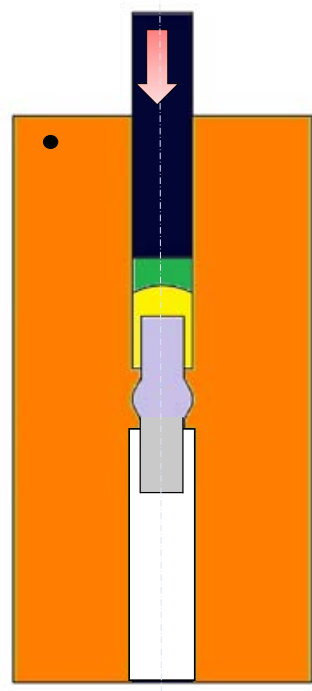

(c)

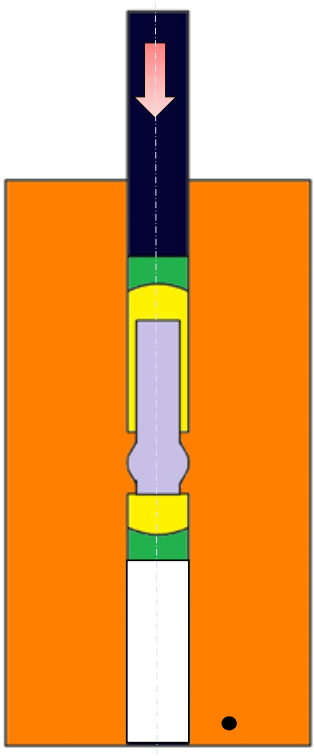

(d)

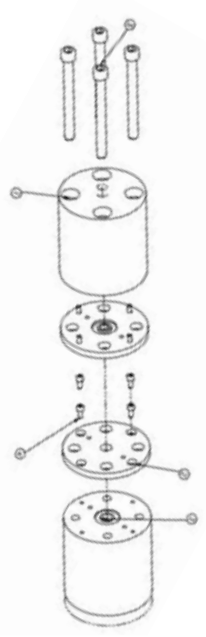

(e)

Fig. 1. Sequences of the HCEE process (a) stage 1, (b) expansion stage, (c) first cycle HCEE processing, and (d) second cycle HCEE processing, (e) die parts.

\section{Result and discussion}

The unprocessed and HCEE processed samples are shown in Fig. 2. As it can be seen, the surface appearance in the processed side of the sample is getting better due to the frictionless characteristic of HCEE process. The maximum length/diameter of the sample which could be produced via conventional CEC or CEE is below 5 [19] while this value was successfully 
selected for HCEE to be 10 as a first trial. However, because the frictionless characteristics of the process, it may be possible to process longer samples via HCEE. The fluid pressure plays a major role in HCEE process, significantly affecting the loading process due to the existence of hydrostatic pressure and eliminating the friction effect [26].

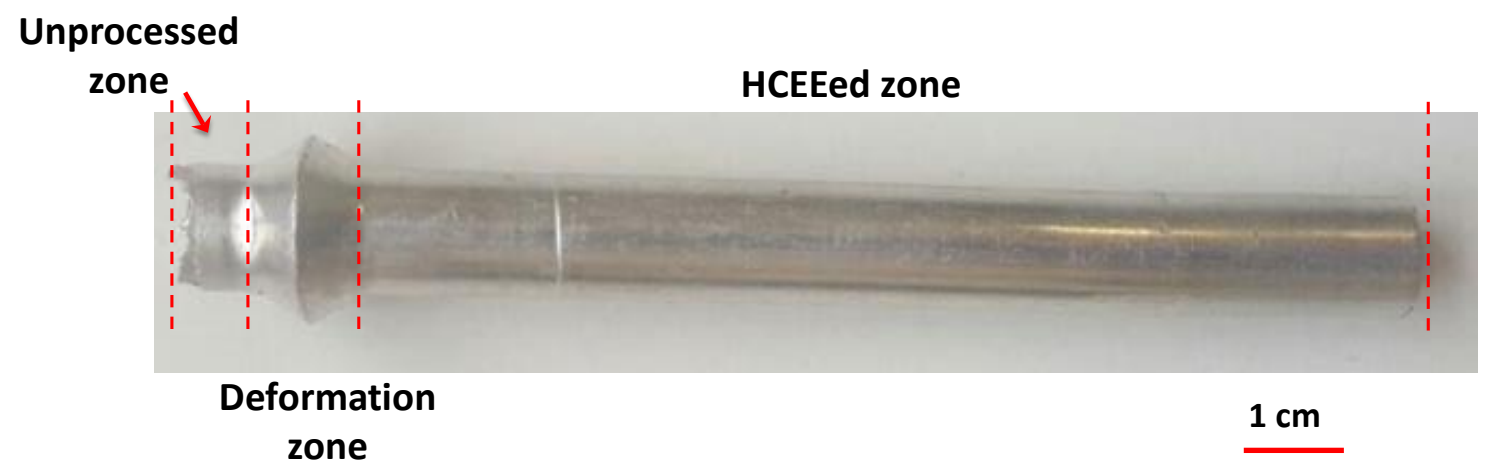

Fig. 2. (a) A workpiece during HCEE processing from the unprocessed zone to HCEE processed zone.

The microstructure of the unprocessed sample is illustrated in the OM micrograph of Fig. 3a, and showing an average grain size of approximately $50 \mu \mathrm{m}$. After performing two passes of HCEE, sub grains was formed and the size of the subgrains decreased to an approximate grain size of $0.76 \mu \mathrm{m}$ at the mid-radius of the cross-section and a perpendicular to the axis direction as shown in the TEM micrograph of Fig. 3b. Large plastic deformation under hydrostatic compressive stresses caused severe reduction of grains and subgrains sizes. At first, geometrical grain shape changed, and subsequently grain subdivision appeared [22, 27]. Two type of regions can be observed in Fig. 3b ( $X$ and $Y)$. The region $X$, which does not have any dislocation and interior of grains, is relatively clean. The region $Y$ contains dislocation tangles and sub-boundaries. As depicted, the grains are elongated, and dislocations are aggregated in some grain boundaries shown by arrows [21, 22]. It seems to have distortion high and low angle boundary's together like previous studies [21, 28] , but the majority of them looks like a low angle boundaries. 


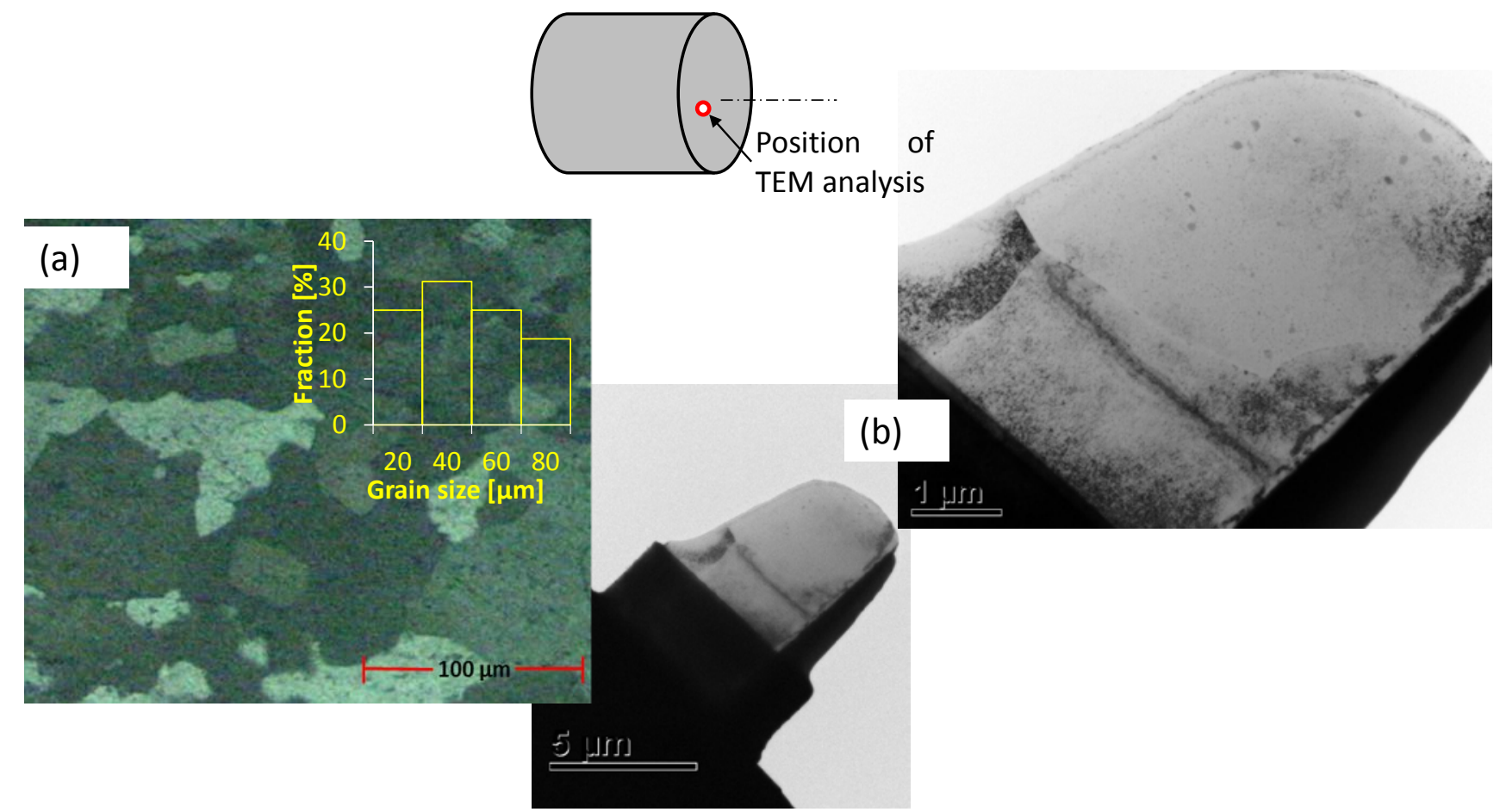

Fig. 3. (a) OM micrograph of the annealed Al 1050 microstructure and (b) TEM micrograph of the two cycles HCEE processed sample.

The distribution of UFG grains is illustrated in Fig. 4 which obtained by electron back scattered diffraction (EBSD) mapping after two HCEE passes. The microstructure shows evidence for grain refinement. The statistical variation of grain size gives an average size of about $0.7 \mu \mathrm{m}$. It is found that more than $50 \%$ of the scanned area has grains less $1 \mu \mathrm{m}$ size. Both elongated and small equiaxed grains appeared in microstructure induced shear bands and large deformation. Small equiaxed grains maybe formed due to continuous recrystallization[29]. The low angle grain boundaries (with misorientation angles between $1^{\circ}$ and $15^{\circ}$ ) are shown by the fine solid lines and the high angle grain boundaries (with misorientation larger than $15^{\circ}$ ) by the solid thick lines. So there are high and low angle boundaries together. The TEM micrograph (Fig. 3b) also showed this result. 

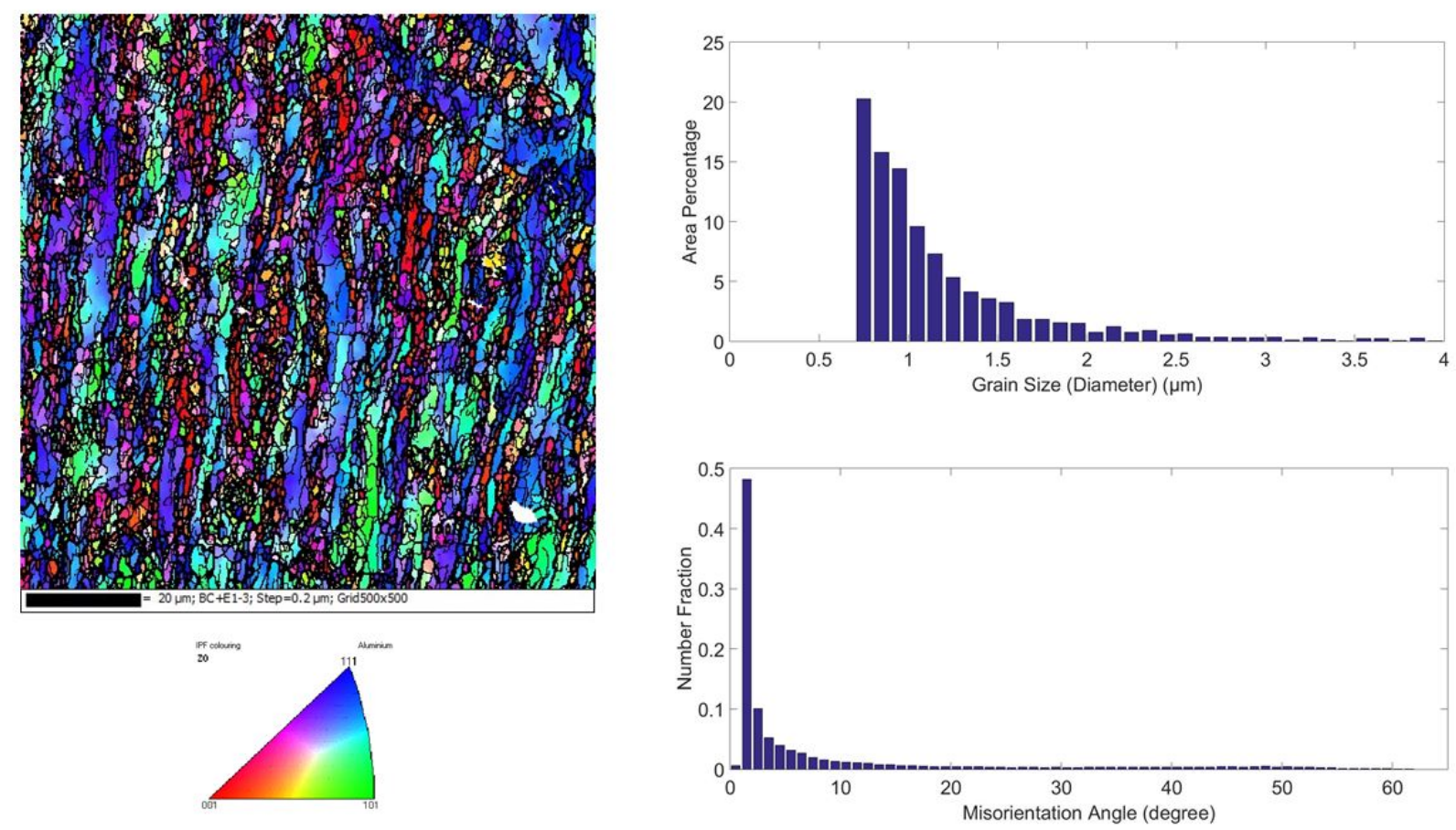

Fig. 4: Inverse pole figure EBSD map of a sample processed to two HCEE passes with distribution of cell size

Fig. 5a illustrates microhardness variation of the unprocessed and HCEE processed sample along the radial direction from the center. The average microhardness of the unprocessed sample was $36 \mathrm{HV}$, and it is increased to $45 \mathrm{HV}$ after the first pass of HCEE process. As depicted, the microhardness value in the center of the sample is less than the outer due to a non-uniform strain distribution achieved from the deformation zone [16]. Fig. 5b shows the tensile stress-strain curves of the unprocessed and HCEE processed samples. As shown, the strength increased considerably after HCEE due to the strain hardening, accumulation of dislocations, and grain refinement following the process. The yield and ultimate strengths for the unprocessed sample were $40 \mathrm{MPa}$ and $52 \mathrm{MPa}$, respectively. They are increased to $109 \mathrm{MPa}$ and $115 \mathrm{MPa}$ just after one pass of HCEE process. After 2 passes, they were increased to $125 \mathrm{MPa}$ and $137 \mathrm{MPa}$, respectively. In Fig. 5, SEM micrographs of the fractured surface of the unprocessed, HCEE processed sample via first and second passes are illustrated. They contain many dimples showing a ductile fracture. These dimples originate from nucleation, growth, and coalescence of microvoids under tensile stresses. As can be seen in Fig. 5c and 3d, after the first and second pass of HCEE process, the diameter of dimples decreases compared to the unprocessed sample 
shown in Fig. 5c which leads to a reduction in the ductility. It can be seen that the fractured surface of the unprocessed specimen contains many dimples distributed like a honeycomb. Furthermore, the depth of the dimples is decreased following an initial pass of the HCEE process. However, it is noted that the dimple features become deeper and smaller as the number of HCEE passes is increased. The size of dimples is usually related to the nucleation sites and the number of voids initiated at the grain boundaries. Thus, a continuous decrease in dimple size of HCEE-processed specimens is likely to be a result of the microstructural refinement that occurred during the SPD process [30].
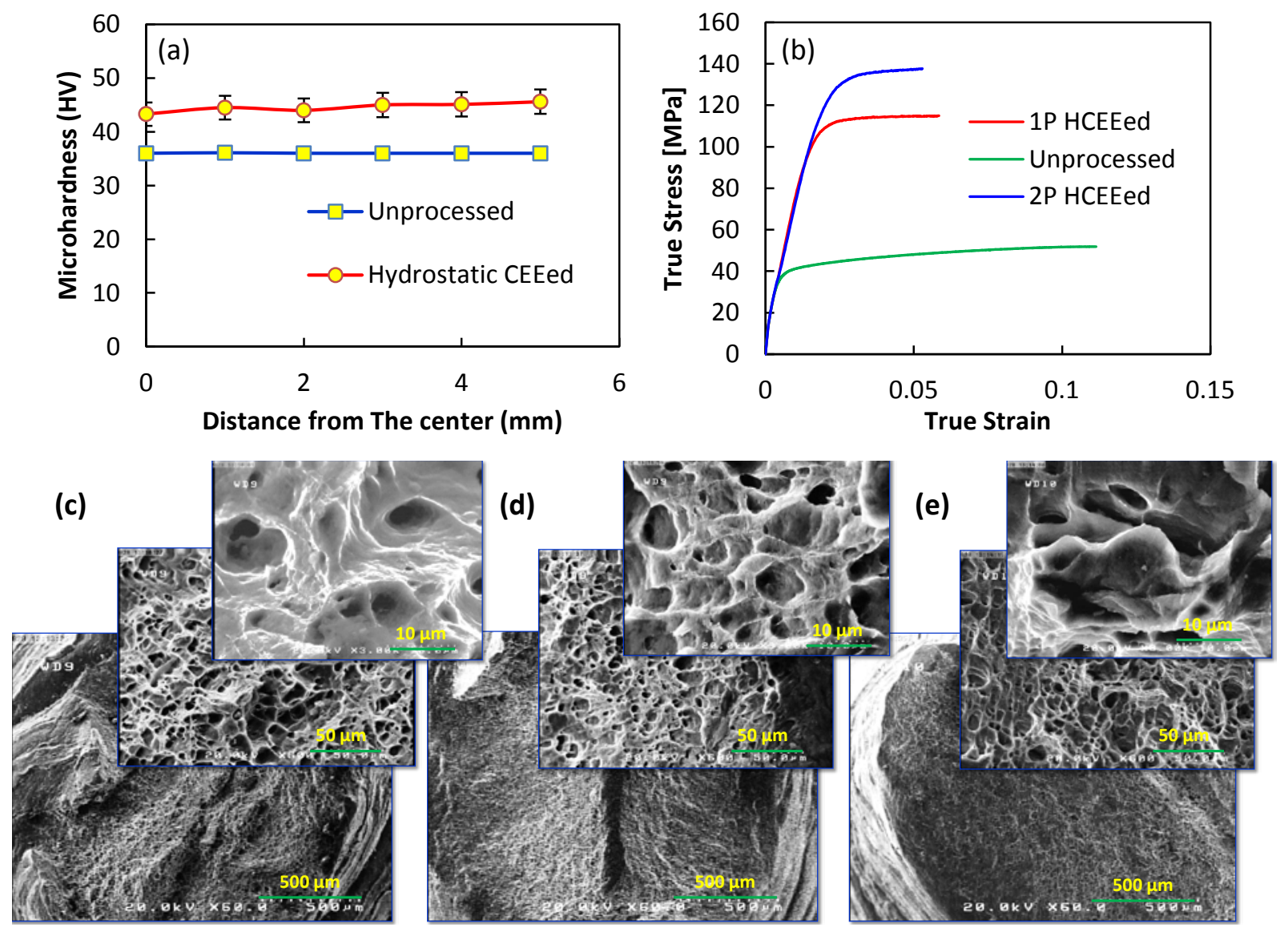

Fig. 5. (a) Microhardness variation of the unprocessed sample and the first pass of HCEE process, (b) True stress-strain curves of unprocessed and HCEE processed, Fractured surfaces after tensile test of (c) unprocessed (d) first pass HCEEed (e) second passes HCEEed specimens. 
Fig. 6a shows force ram movement curves for two processes of conventional CEE and HCEE calculated using FE method. It is seen that the required force for HCEE process (22 $\mathrm{kN})$ is dramatically lower than that for conventional CEE $(180 \mathrm{kN})$. The required force in HCEE is about 89 \% lower than that in CEE. This could be considered as a main advantage of HCEE over conventional CEE. In other words, the required force in HCEE is independent of sample length, and it may be noted that HCEE could be a modern industrial method for processing of large UFG and NG samples. Also, due to reduced friction force by progressing the punch, the total force in CEE process decreases after a peak. However, in HCEE process the total force is steady because the friction force is approximately zero. Fig. 6b shows a magnified FE estimated force-ram displacement curve of HCEE. The force curve can be divided into two sections. In the first section relating to Fig. 1b, where the expansion zone of the die fills and the force is increased sharply to $19 \mathrm{kN}$. In the second section, (where the bottom punch of Fig. 1 is removed) the extrusion process force is increased slightly, and after the sample starts to flow, the force decreases and will be consistent up to the end of the process. Fluctuations of the force curve are because of discontinuous contact between the sample and die in FE method.

The amount of equivalent plastic strain after one pass of HCEE process can be calculated from Eq. 1 [16] as $\tilde{\varepsilon} \approx 1.34$ though the distribution of strain is almost complex in reality [31]. Fig. 6(c) and (d) show the contour and path plot of equivalent plastic strain along the cross section of the HCEE processed sample. Strain distribution along the length of the sample is approximately uniform but it is non-uniform along the radial direction. Fig. 6d shows the distribution of equivalent strain measured by FEM and the theory calculated along the radial direction of the sample. The average equivalent strain in the FEM (1.309) is close to the theoretical value (1.34), but its distribution is highly complex. The strain distribution in the FEM with a microhardness distribution in experimental state was compared in Fig6. The distribution of microhardness was similar to the nonuniform strain distribution, However, the difference in the value of the microhardness is not as large as the difference in the amount of strain across the sample. Because the hardness depends on a large number of parameters, such as texture, grain and sugrain size as well as the distribution of dislocation[32]. According to the previous content, the results of FEM were in good agreement with the theoretical equation and exprimental technique. Also, simulation results are close to the results of the CEE method[16]. 

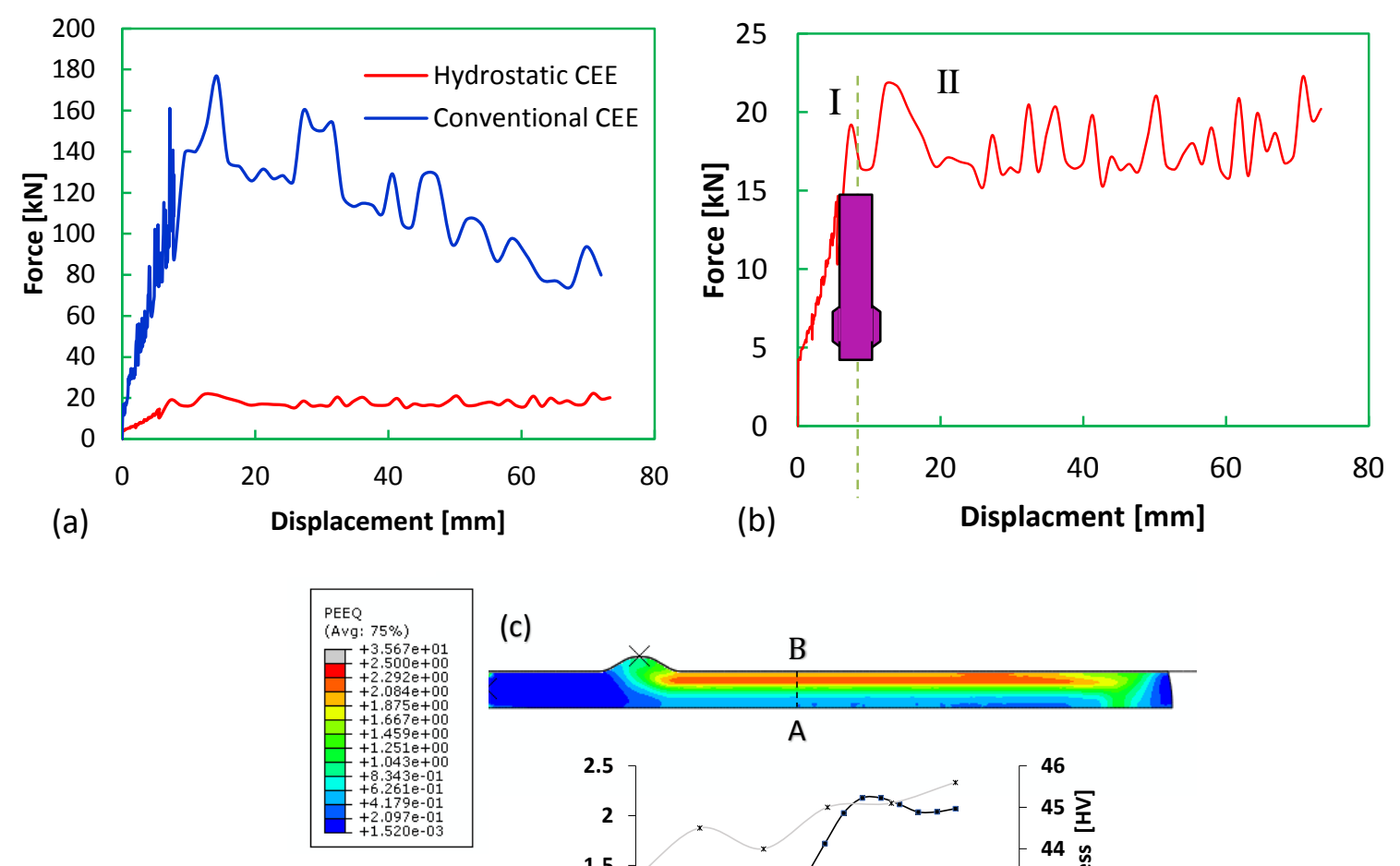

(c)
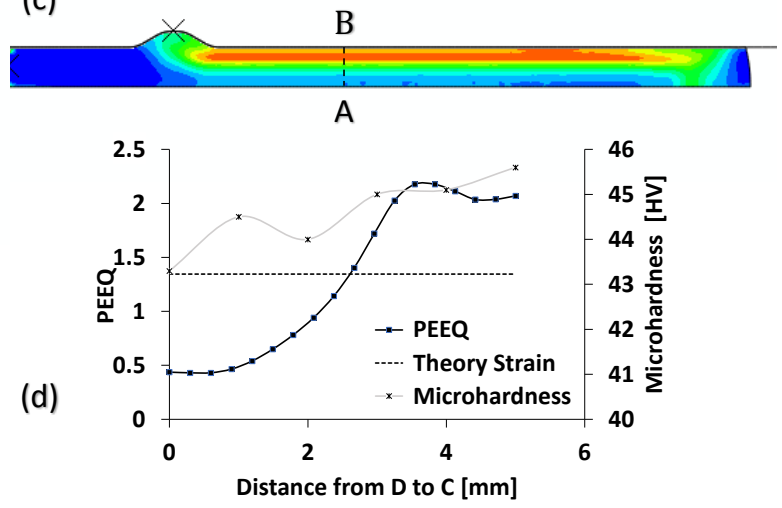

Fig. 6. (a) FEM calculated force versus ram displacement during the HCEE and CEE processes, (b) Magnified force-displacement curve during the HCEE process, (c) and (d) Contour and path plot of equivalent plastic strain along the cross-section of the HCEE processed sample.

\section{Conclusion}

A new method entitled Hydrostatic Cyclic Expansion Extrusion is developed based on the conventional CEE. The most important advantage of this new technique compared to conventional CEE is the ability to process samples regardless of length, because the process is frictionless and there is no contact between the samples and die. The process was performed and analyzed, with several promising conclusions being observed.

- HCEE could process metals with a good surface appearance.

- Because of approximately zero friction, HCEE process is almost independent of sample length, and longer samples may be easily processed. 
- Significant grain refinement was achieved, and the subgrain size decreased to $\sim 760 \mathrm{~nm}$ from the initial value of $\sim 50 \mu \mathrm{m}$.

- Yield and ultimate strength increased considerably to 109 and 115 from the initial values of $40 \mathrm{MPa}$ and $52 \mathrm{MPa}$, respectively.

- The microhardness of samples after two passes of HCEE process improved from 36 to 45 HV.

- The process load decreased by approximately 89\% in HCEE compared to conventional CEE

- , the results of FEM were in good agreement with the theory equation and experimental method

\section{Acknowledgements}

This work has been supported by Iran National Science Foundation (INSF).

\section{References}

[1] G. Faraji, H. Kim, Materials Science and Technology, 33 (2017) 905-923.

[2] T.G. Langdon, Acta Materialia, 61 (2013) 7035-7059.

[3] V. Segal, Materials Science and Engineering: A, 197 (1995) 157-164.

[4] S.N. Alhajeri, N. Gao, T.G. Langdon, Materials Science and Engineering: A, 528 (2011) 3833-3840.

[5] J. Zhang, in: Materials Science Forum, Trans Tech Publ, 2007, pp. 2293-2300.

[6] N. Takata, S.-H. Lee, N. Tsuji, Materials Letters, 63 (2009) 1757-1760.

[7] M. Eskandarzade, A. Masoumi, G. Faraji, M. Mohammadpour, X.S. Yan, Journal of Alloys and Compounds, 695 (2017) 1539-1546.

[8] J. Moon, Y. Qi, E. Tabachnikova, Y. Estrin, W.-M. Choi, S.-H. Joo, B.-J. Lee, A. Podolskiy, M. Tikhonovsky, H.S. Kim, Materials Letters, 202 (2017) 86-88.

[9] M.R. J. Richert, Aluminum, 62 (1986) 604-607.

[10] J.-W. Yeh, S.-Y. Yuan, C.-H. Peng, Metallurgical and Materials Transactions A, 30 (1999) 2503-2512.

[11] T.-C. Chang, J.-Y. Wang, C.-M. O, S. Lee, Journal of Materials Processing Technology, 140 (2003) 588591.

[12] M. Richert, Q. Liu, N. Hansen, Materials Science and Engineering: A, 260 (1999) 275-283.

[13] S.-W. Lee, J.-W. Yeh, Metallurgical and Materials Transactions A, 36 (2005) 2225-2234.

[14] J. Lin, Q. Wang, L. Peng, H.J. Roven, Journal of Alloys and Compounds, 476 (2009) 441-445.

[15] Y.J. Chen, Q.D. Wang, H.J. Roven, M.P. Liu, M. Karlsen, Y.D. Yu, J. Hjelen, Scripta Materialia, 58 (2008) 311-314.

[16] N. Pardis, B. Talebanpour, R. Ebrahimi, S. Zomorodian, Materials Science and Engineering: A, 528 (2011) 7537-7540.

[17] J. Richert, M. Richert, M. Mroczkowski, International Journal of Material Forming, 1 (2008) 479-482.

[18] M.W. Richert, in: Solid state phenomena, Trans Tech Publ, 2006, pp. 19-28.

[19] S. Amani, G. Faraji, K. Abrinia, Journal of Manufacturing Processes, 28 (2017) 197-208. 
[20] S. Amani, G. Faraji, H. Kazemi Mehrabadi, K. Abrinia, H. Ghanbari, Journal of Alloys and Compounds. [21] M. Lewandowska, in: Solid State Phenomena, Trans Tech Publ, 2006, pp. 109-116.

[22] M. Lewandowska, K.J. Kurzydlowski, Journal of Materials Science, 43 (2008) 7299.

[23] F.S. G.Faraji, P. Babaei, IR-Patent (in persian), 139550140003008482 (2017).

[24] F. Rahimi, A. Eivani, Materials Science and Engineering: A, 626 (2015) 423-431.

[25] J. Lv, F. Hu, Q.D. Cao, R. Yuan, Z. Wu, H. Cai, L. Zhao, X. Zhang, Journal of Materials Engineering and Performance, 26 (2017) 1463-1474.

[26] S. Jamali, G. Faraji, K. Abrinia, The International Journal of Advanced Manufacturing Technology, 88 (2017) 291-301.

[27] M.J. Zehetbauer, Y.T. Zhu, Bulk nanostructured materials, John Wiley \& Sons, 2009.

[28] P.-L. Sun, P.-W. Kao, C.-P. Chang, Metallurgical and Materials Transactions A, 35 (2004) 1359-1368.

[29] M. Mesbah, G. Faraji, A. Bushroa, Metals and Materials International, 22 (2016) 288.

[30] M. Montazeri-Pour, M.H. Parsa, Mechanics of Materials, 94 (2016) 117-131.

[31] S. Amani, G. Faraji, K. Abrinia, Journal of Manufacturing Processes, (2017).

[32] P. Hidalgo, C. Cepeda-Jiménez, O. Ruano, F. Carreño, in: Materials Science Forum, Trans Tech Publ, 2010, pp. 1929-1933. 